\title{
Further phenotypic delineation of the auriculocondylar syndrome type 2 with literature review
}

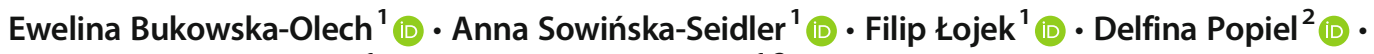 \\ Joanna Walczak-Sztulpa ${ }^{1}$ (D) $\cdot$ Aleksander Jamsheer ${ }^{1,2}$ (I)
}

Received: 16 August 2020 / Revised: 15 October 2020 / Accepted: 21 October 2020 / Published online: 31 October 2020

(C) The Author(s) 2020, corrected publication 2020

\begin{abstract}
Auriculocondylar syndrome (ACS) is an ultra-rare disorder that arises from developmental defects of the first and second pharyngeal arches. Three subtypes of ACS have been described so far, i.e., ACS1 (MIM: 602483), ACS2 (MIM: 600810), and ACS3 (MIM: 131240). The majority of patients, however, are affected by ACS2, which results from the mutations in the PLCB4 gene. Herein, we have described an 8-year-old male patient presenting with ACS2 and summarized the molecular and phenotypic spectrum of the syndrome. We have also compared the clinical features of our case to three other previously described cases (one sporadic and two familial) harboring the same heterozygous missense variant c.1862G>A, p.Arg621His in the PLCB4 gene. The mutation was detected using whole-exome sequencing (WES). Due to low coverage of WES and suspicion of somatic mosaicism, the variant was additionally reassessed by deep targeted next-generation sequencing panel of genes related to the craniofacial disorders, and next confirmed by Sanger sequencing. ACS2 presents high intra- and interfamilial phenotypic heterogeneity that impedes reaching an exact clinical and molecular diagnosis. Thus, describing additional cases, carrying even the known mutation, but resulting in variable phenotypes, is essential for better understanding of such orphan Mendelian diseases.
\end{abstract}

Keywords Next-generation sequencing · PLCB4 $\cdot$ Question mark ear · Auriculocondylar syndrome

\section{Introduction}

Auriculocondylar syndrome (ACS), also known as question mark ear syndrome or dysgnathia complex, is an ultra-rare disorder that belongs to a group of malformations arising from the developmental defects of the first and second pharyngeal arches (Passos-Bueno et al. 2009). Three subtypes of ACS have been described so far, i.e., ACS1 (MIM: 602483), ACS2 (MIM: 600810), and ACS3 (MIM: 131240), resulting

Communicated by: Michal Witt

Supplementary Information The online version contains supplementary material available at https://doi.org/10.1007/s13353-02000591-3.

Aleksander Jamsheer

jamsheer@wp.pl

1 Department of Medical Genetics, Poznan University of Medical Sciences, Rokietnicka 8 Street, 60-806 Poznan, Poland

2 Centers for Medical Genetics GENESIS, Dąbrowskiego 77A Street, 60-529 Poznan, Poland from deleterious variants within GNAI3 encoding G protein subunit alpha i3, PLCB4 encoding phospholipase $\mathrm{C}$ beta 4 , and $E D N 1$ encoding endothelin 1, respectively (Rieder et al. 2012; Gordon et al. 2013a, b). Noteworthy, all of those genes constitute the EDN1-EDNRA pathway, which contributes to craniofacial development through induction, migration, and maintenance of neural crest cells (Bonano et al. 2008).

The clinical diagnosis of ACS may be challenging due to its high intra- and interfamilial phenotypic variability and infrequent occurrence, which is estimated to be $<1$ per $1,000,000$ (Nabil et al. 2020; Storm et al. 2005). However, the core features include the variable degree of micrognathia, often associated by temporomandibular joint ankylosis, cleft palate, and distinctive ear malformations in the form of cleft or notch within the helix, giving the appearance of a question mark. Other frequently noted features comprise facial asymmetry, hearing impairment, prominent cheeks, preauricular tags, and microstomia (Gordon et al. 2015; Romanelli Tavares et al. 2017).

Herein, we report the next case of ACS2 and summarize the molecular and phenotypic spectrum of the syndrome. We 
have also compared the clinical features of an 8-year-old patient to three other previously described cases (one sporadic and two familial), harboring the same heterozygous missense variant c. $1862 \mathrm{G}>\mathrm{A}, \mathrm{p} . \mathrm{Arg} 621 \mathrm{His}$ in the PLCB4 gene. The mutation was detected using whole-exome sequencing (WES). Due to low coverage and suspicion of somatic mosaicism in WES analysis, the variant was additionally assessed by deep targeted next-generation sequencing (NGS), and confirmed by Sanger sequencing.

\section{Methods}

\section{GTG banding and microarray analysis}

We performed karyotyping on peripheral blood lymphocytes of the index patient using the Giemsa-banding technique at 550 band resolution per haploid genome. Moreover, array comparative genomic hybridization (aCGH) (SurePrint G3 Human CGH Microarray $8 \times 60 \mathrm{k}$; Agilent Technologies) was performed on the high-quality genomic DNA (gDNA) of the index. gDNA was extracted from the peripheral blood lymphocytes using the MagCore ${ }^{\circledR}$ HF16 automated nucleic acid extractor (RBC Bioscience Corp.).

\section{Whole-exome sequencing}

Whole-exome sequencing (WES) was performed in the proband and his healthy parents using the Sure Select Enrichment Kit (Agilent Technologies) and Illumina Paired-End Sequencing Kit (Agilent Technologies) followed by sequencing on Illumina HiSeq 1500. The sequenced reads were mapped to the GRCh38 human reference genome. A variant discovery pipeline was built based on the GATK Best Practices. In order to extract potentially causative variants, we applied a Phenotypic Interpretation of eXomes (PhenIX) computational algorithm using the following Human Phenotype Ontology (HPO) terms: micrognathia (HP: 0000347), atresia of the external auditory canal (HP: 0000413), and microtia (HP: 0008551) (Jamsheer et al. 2016).

\section{Targeted next-generation sequencing}

We used the custom On-Demand AmpliSeq (Thermo Fisher Scientific) gene panel with 2 primer pools and sequenced 37 genes related to the craniofacial disorders, including PLCB4 (Supplementary File 1) (Bukowska-Olech et al. 2020). The barcoded gDNA libraries were constructed according to the manufacturer's sample preparation protocol (Ion AmpliSeq Library Kit 2.0; On-Demand Panels) and subsequently sequenced on the Ion Torrent S5 platform with the use of the Ion $540^{\mathrm{TM}} \mathrm{Kit}$. The sequenced reads were mapped to the
GRCh38 human reference genome, and the variant of interest (chr20:9389727) was analyzed applying the Integrative Genomics Visualization (IGV) tool.

\section{Sanger sequencing}

We performed the validation of the variant using PCR and conventional Sanger sequencing. Specific primers were designed with the use of the Primer3 tool v. 0.4.0 (http:// bioinfo.ut.ee/primer3-0.4.0/). Primer sequences $5^{\prime}-3^{\prime}$ were as follows: gctgtgacaatctgcccaaa (forward), ggcaccatcctgtcaaagtg (reverse). The PCR and PCR product purification were carried out following standard protocols. Sanger sequencing was performed on the automated sequencer Applied Biosystems Prism 3700 DNA Analyser using the dye-terminator chemistry kit v.3, ABI 3130XL. Next, the variant was visualized by the BioEdit tool and annotated against the reference PLCB4 sequence NM_000933.3 following the Human Genome Variation Society (HGVS) nomenclature guidelines.

\section{In silico analysis}

The 3D structure of the PLCB4 mutation site was predicted using the SWISS-MODEL homology modeling server (Waterhouse et al. 2018). As a template, the rat phosphoinositide-specific phospholipase $\mathrm{C}$, isozyme delta1 (1djz.2.A) structure was used (Essen et al. 1997). The template had a $37.67 \%$ sequence homology with the human PLCB4, had an X-ray crystal structure of high resolution (3.0 ̊), and contained 1D-myo-inositol-4,5-bisphosphate (IP2) ligand. The modeling was performed for the wild-type and mutated protein. Conservation analysis was done applying the Varsome tool (https://varsome.com/) and Alamut ${ }^{\circledR}$ Visual software.

\section{Clinical report}

The 8-year-old male patient was born to unrelated and healthy parents in the $40^{\text {th }}$ week of gestation by Cesarean section from the $5^{\text {th }}$ pregnancy complicated with threatened abortion. His body mass was $2860 \mathrm{~g}\left(10^{\text {th }}\right.$ percentile $)$, length $53 \mathrm{~cm}\left(75^{\text {th }}\right.$ $90^{\text {th }}$ percentile), and head circumference $31 \mathrm{~cm}$ (below $3^{\text {rd }}$ percentile), while the Apgar score was 10 at $1^{\prime}$. After birth, marked mandibular hypoplasia (micro- and retrognathia), facial asymmetry, atresia of the right external auditory canal, and asymmetric hypoplasia of both pinnae (more severe on the right side) were observed. Psychomotor development was within normal range, with independent sitting achieved at 6 months, and walking at 18 months of age. The patient spoke first words at 12 months of age, but further speech development was delayed due to conductive hypoacusis. During the last clinical evaluation at the age of 8 years, the index had normal intellectual development, but manifested aggressive 
behavior. His abdominal ultrasound scan was inconspicuous. The main clinical features described during the genetic evaluation are listed in Table 1 and Fig. 1.

\section{Results}

\section{GTG banding and microarray analysis}

Conventional chromosome analysis (GTG banding) based on the patient's peripheral blood lymphocytes showed normal male karyotype. Microarray analysis with the use of $60 \mathrm{k} 8$ plex array (SurePrint G3 Human CGH, Agilent) detected no copy number variations larger than $100 \mathrm{~kb}$ in proband's whole-blood DNA.

\section{Next-generation and Sanger sequencing methods}

WES revealed that the affected individual carried the heterozygous variant c. $1862 \mathrm{G}>\mathrm{A}$, p. Arg621His in the $20^{\text {th }}$ exon of PLCB4 (hg38; chr20:9409080, RefSeq NM_000933.3), whereas both of his healthy parents were negative for this variant. The missense mutation was reported in the Human Gene Mutation Database (HGMD; CM123396), Online Mendelian Inheritance in Man (OMIM; 600810.0003), ClinVar (accession: VCV000031639.1; variation ID: 31639), and dbSNP (rs387514481) databases. Due to low coverage and suspicion of somatic mosaicism in WES, the alteration was additionally assessed by deep targeted nextgeneration sequencing. In this step, we achieved 2177 reads for the analyzed variant. Our result demonstrated heterozygosity of the mutation since the reference adenine nucleotide was called in 1070 reads, whereas mutated guanine in 1096 reads. The alteration was subsequently confirmed and shown to be heterozygous by Sanger sequencing (Fig. 2).

\section{D modeling of the PLCB4 protein mutation site and conservation analysis}

The 3D visualization of wild-type (WT) and mutated (Arg621His) structures of the PLCB4 active site revealed the lack of interaction with IP2 in the mutated protein as compared

Table 1 The comparative analysis of clinical features present in the index case vs. reported patients with auriculocondylar syndrome 2 caused by c.1862G $>$ A, p.Arg621His PLCB4 alteration

\begin{tabular}{|c|c|c|c|c|c|c|c|}
\hline & $\begin{array}{l}\text { Index } \\
\text { case }\end{array}$ & M001 & Case 4 & $\begin{array}{l}\text { Family } 1 \\
\text { Case III: } 2\end{array}$ & $\begin{array}{l}\text { Family } 1 \\
\text { Case II: } 4\end{array}$ & $\begin{array}{l}\text { Family } 1 \\
\text { Case III: } 1\end{array}$ & $\begin{array}{l}\text { Family } 1 \\
\text { Case II: } 1\end{array}$ \\
\hline Submitter & & Rieder et al. & Gordon et al. & Nabil et al. & Nabil et al. & Nabil et al. & Nabil et al. \\
\hline Occurrence & Sporadic & Familial & Sporadic & Familial & Familial & Familial & Familial \\
\hline Gender & M & M & $\mathrm{M}$ & $\mathrm{F}$ & M & M & M \\
\hline $\begin{array}{l}\text { Micrognathia and/ or } \\
\text { retrognathia }\end{array}$ & + & + & + & + & + & + & + \\
\hline Ankylosis & - & - & $?$ & + & $?$ & $?$ & + \\
\hline Cleft palate & - & - & - & - & - & - & - \\
\hline Ear malformation & + & + & + & + & + & + & + \\
\hline Prominent cheeks & + & + & + & + & + & + & + \\
\hline Auricular tags & - & + & + & - & - & - & - \\
\hline Microstomia & + & + & + & + & - & - & + \\
\hline Mastication difficulties & + & + & + & + & - & - & + \\
\hline $\begin{array}{l}\text { Speech articulation } \\
\text { difficulties }\end{array}$ & + & $?$ & + & + & - & - & + \\
\hline Obstructive apnea & - & $?$ & $?$ & + & - & - & + \\
\hline Snoring & + & $?$ & $?$ & + & - & - & + \\
\hline Crowded teeth & + & $?$ & - & + & - & + & - \\
\hline Round face & + & + & + & + & + & - & + \\
\hline Facial asymmetry & + & + & + & + & + & + & + \\
\hline $\begin{array}{l}\text { Mandibular condyle } \\
\text { hypoplasia }\end{array}$ & + & $?$ & + & + & $?$ & $?$ & + \\
\hline Asymmetric mandible & + & + & + & + & + & + & + \\
\hline Short mandibular rami & + & $?$ & + & + & $?$ & + & + \\
\hline Additional feature & - & $\begin{array}{l}\text { Absence of } \\
\text { facial hair }\end{array}$ & $\begin{array}{l}\text { Neonatal hypotonia, } \\
\text { developmental delay }\end{array}$ & $\begin{array}{l}\text { Unilateral } \\
\text { thymic cyst }\end{array}$ & $\begin{array}{l}\text { Absence of } \\
\text { facial hair }\end{array}$ & $\begin{array}{l}\text { Absence of } \\
\text { facial hair }\end{array}$ & $\begin{array}{l}\text { Right neck } \\
\text { lesion }\end{array}$ \\
\hline
\end{tabular}




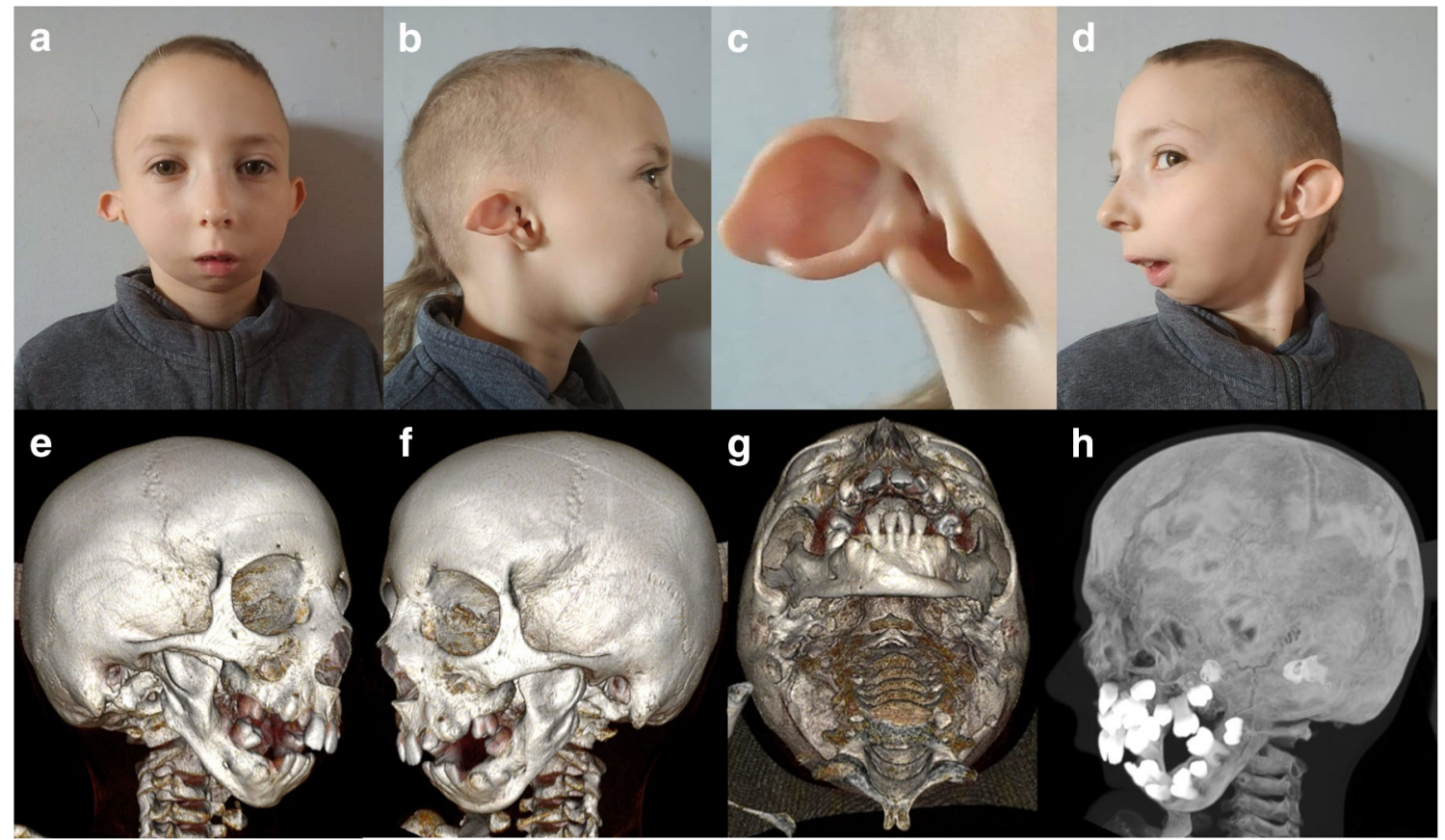

Fig. 1 Phenotypic presentation of the index patient. a Anterior posterior view of the face showing full cheeks and round facial shape. $\mathbf{b}, \mathbf{d}$ Lateral view of the face. $\mathbf{b}, \mathbf{c}$ The notch within the right helix, giving the appearance of a question mark ear. $\mathbf{e}-\mathbf{h}$ A $3 \mathrm{D}$ reconstruction of a computational tomography indicating micrognathia, with mandibular

hypoplasia more pronounced on the left side. Furthermore, coronoid processes and mandibular condyles are significantly hypoplastic on both sides. Lateral view of the patient's skull shows crowded teeth in the upper and lower jaws

to the native molecule (Fig. 3b) (Hicks et al. 2008). Besides, we have shown the conservation of the p.Arg621 residue among eleven species and through bioinformatics scores such as the

PhastCons100way (dbNSFP v4.0) score in vertebrate-1, PhyloP100way (dbNSFP v4.0) score in vertebrate-10.003, and Genomic Evolutionary Rate Profiling (GERP) score 6.17.

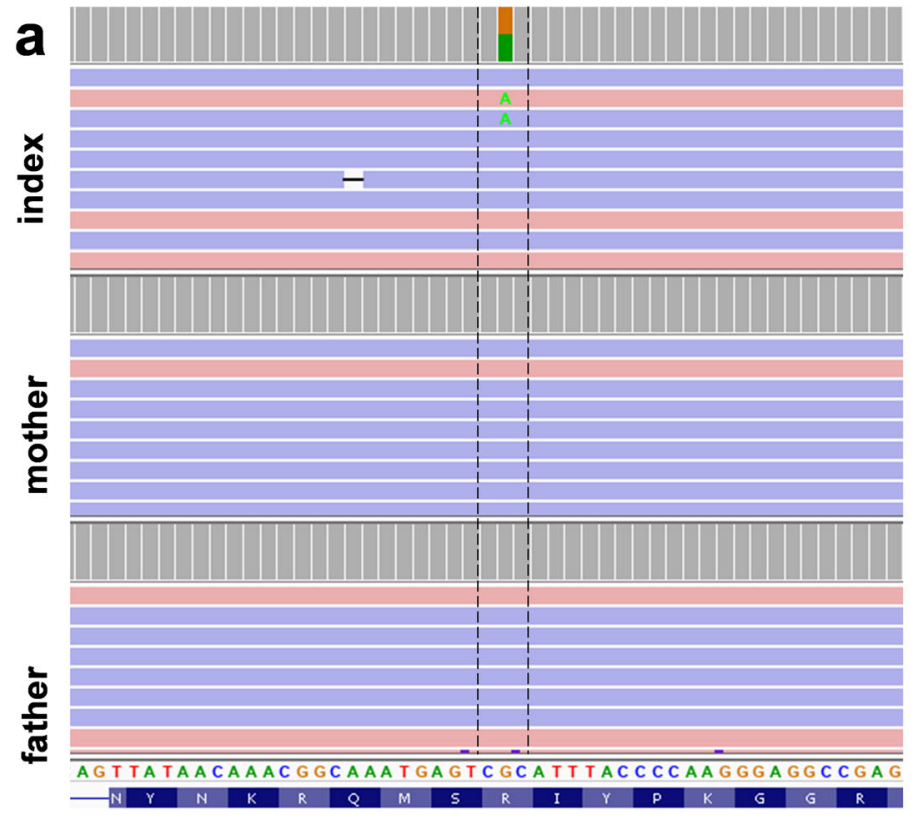

b $\quad$ G

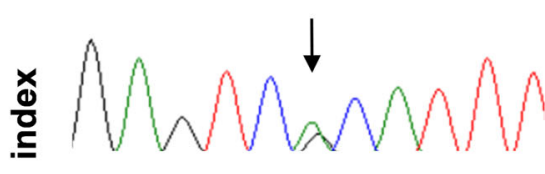

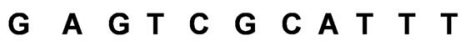

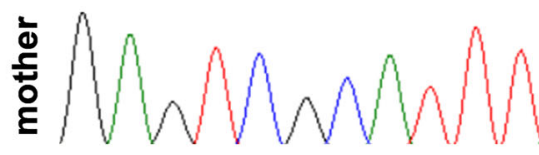

G A G T C G C A T T T T

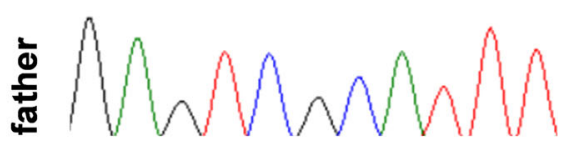

Fig. 2 The molecular results in the index patient. The representation of the c. $1862 \mathrm{G}>\mathrm{A} P L C B 4$ variant revealed in heterozygosity in the index (a - upper image) and excluded in both parents (a-mother \& father in the middle and bottom image, respectively) employing targeted next- generation sequencing. Chromatograms show targeted validation studies of the index patient (b - upper image) and his parents (b - middle and bottom images) performed with the use of Sanger sequencing 
Fig. 3 a The PLCB4 protein structure. Schematic representation of the PLCB4 protein with marked mutations reported in ACS2. PI-PLC X-box - phosphatidylinositol-specific phospholipase C X domain; PIPLC Y-box - phosphatidylinositol-specific phospholipase C Y domain; C2 - C2 domain. b 3D structure prediction of the active site of wild-type (WT) and mutated (Arg621His) PLCB4. Hydrogen bonds and salt bridges between amino acid residues and 1D-myo-inositol-4,5bisphosphate (IP2) are shown as blue and yellow dashed lines, respectively. Red and blue colors indicate negatively and positively charged amino acids. Note, lack of interaction with IP2 in the mutated (Arg621His) protein
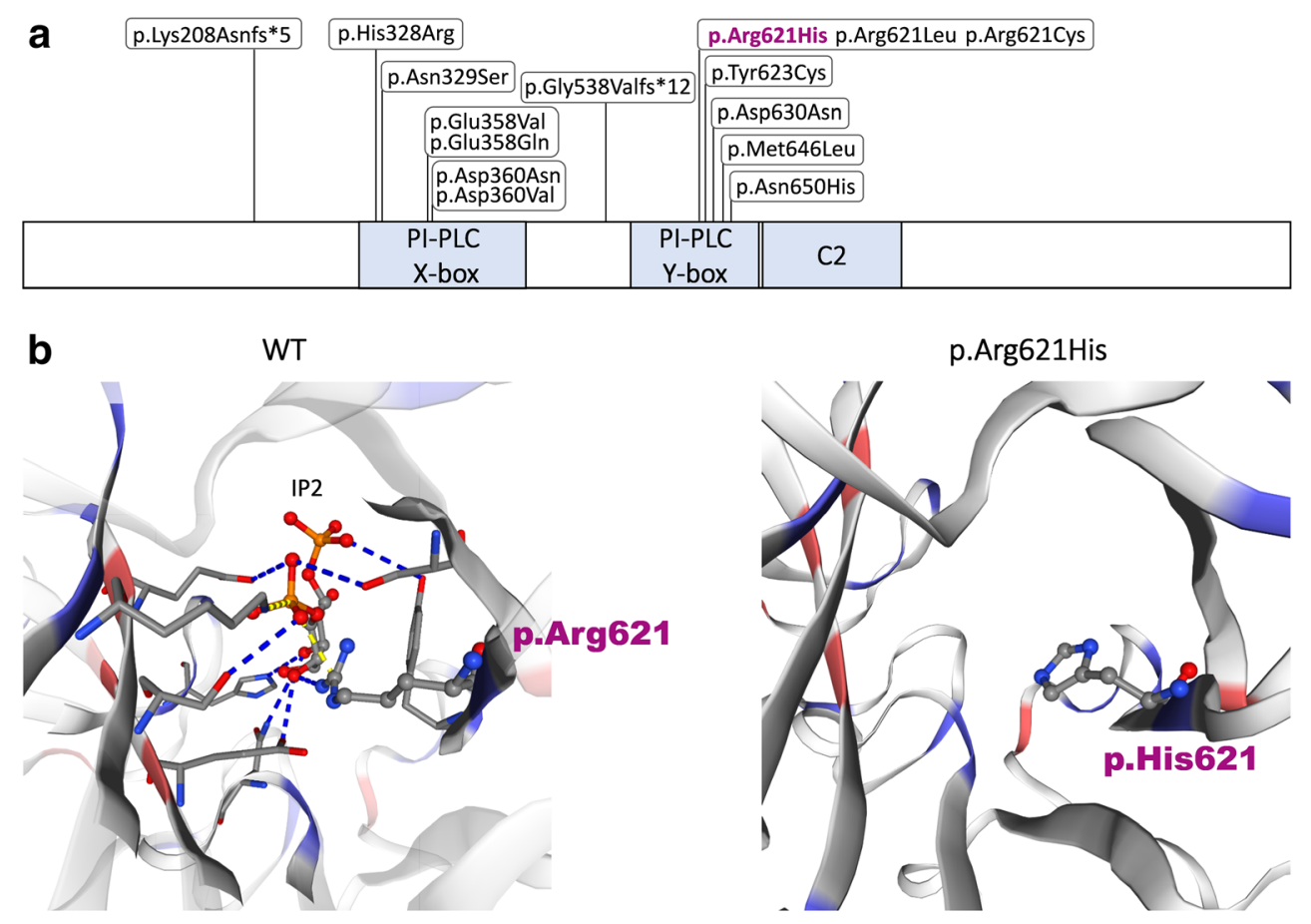

\section{Discussion}

In the current study, we are describing another individual presenting with ACS2, which is an ultra-rare disease. The 8-yearold boy is the $4^{\text {th }}$ reported patient carrying the missense mutation p.Arg621His in the PLCB4 gene, in addition to case M001 described by Rieder et al., case 6 by Gordon et al., and the familial cases III: 2, II: 4, III: 1 , and II: 1 by Nabil et al. (Table 1) (Rieder et al. 2012; Gordon et al. 2013a, b; Nabil et al. 2020). We obtained the molecular diagnosis applying the trio WES approach. Since the coverage of PLCB4 was only $80 \mathrm{x}$ and results indicated a possibility of somatic mosaicism in the index, we subjected the patient's and his parents' DNA to deep targeted NGS of a custom craniofacial disorders-associated gene panel (Supplementary File 1) with a mean coverage of 2000x. The data we have obtained, however, pointed to the heterozygosity of the p.Arg621His missense variant, which was further confirmed through Sanger sequencing (Fig. 2).

Interestingly, the alteration, which we have described, was the first causative mutation linked to ACS2 (Rieder et al. 2012). The clinical features resulting from this variant have been shown in Table 1. All patients present with micrognathia, prominent cheeks, facial asymmetry, asymmetric mandible, and question mark ears. Those features seem to be cardinal for ACS. Notably, none of the affected individuals had either a cleft palate or a hearing impairment. Three individuals, however, displayed obstructive apnea, which has been described as an unusual feature of ACS2. Another interesting finding is the presence of microstomia and full mouth at the same time
(Table 1; cases III: 2 and II: 4 vs. cases III: 1 and II: 1). The clinical spectrum of this Mendelian disease is heterogeneous and may overlap with other syndromes that result from the developmental defects of the first and second pharyngeal arches. Hence, the precise dysmorphological assessment in those patients constitutes a crucial stage of genetic counseling.

ACS2 inherits in an autosomal dominant manner and has been linked to thirteen heterozygous missense mutations in the PLCB4 gene, so far. All those point variants occur within highly conserved catalytical $\mathrm{X}$ and $\mathrm{Y}$ domains of phospholipase $\mathrm{C}$ beta 4 protein (Fig. 3a) (Leoni et al. 2016; Rieder et al. 2012; Gordon et al. 2013a, b; Romanelli Tavares et al. 2017; Kido et al. 2013). However, three exceptional recessively inherited cases (two sporadic and one familial), presenting with a more severe phenotype and additional atypical symptoms, have been reported in the medical literature. First, Gordon et al. described an individual carrying a homozygous PLCB4 alteration-c.1612-279_2015+1546del14997; p.Gly538Val $f * 12$ - that was inherited from the healthy consanguineous heterozygous parents (Gordon et al. 2013a, b). Second, Kido et al. presented two siblings being compound heterozygotes for c. $854-1 \mathrm{G}>\mathrm{A}$ and c. $1238+1 \mathrm{G}>\mathrm{C}$ variants, inherited from the healthy non-consanguineous father and mother, respectively (Kido et al. 2013). Finally, the third patient, who was born to a healthy, consanguineous couple, harbored a homozygous deletion c.624delG; p.(Lys208Asn $\left.f s^{*} 5\right)$ (Leoni et al. 2016). Noteworthy, none of the stop gain, frameshift, splicing, and deletion type mutations was identified in heterozygosity among ACS2 cases. Screening of the gnomAD database, however, showed the presence of this type 
Table 2 An overview of stop gain and frameshift variants found in the PLCB4 gene available in gnomAD browser database (accessed on 27 July 2020). Interestingly, one missense variant p.Arg621Cys associated with ACS2 was reported with the populational frequency of 0.000319

\begin{tabular}{|c|c|c|c|}
\hline Variant ID & Consequence & Annotation & Allele frequency \\
\hline 20-9389726-C-T & p.Arg621Cys & Missense & $3.19 \mathrm{E}-05$ \\
\hline 20-9404500-C-T & p.Arg797* & Stop gained & $3.98 \mathrm{E}-06$ \\
\hline 20-9404515-C-T & p.Arg802* & Stop gained & $3.98 \mathrm{E}-06$ \\
\hline 20-9404524-G-T & p.Gly $805^{*}$ & Stop gained & $3.98 \mathrm{E}-06$ \\
\hline 20-9417677-CT-C & p.Ser870Pro $f s^{*} 16$ & Frameshift & $3.98 \mathrm{E}-06$ \\
\hline 20-9440284-CAA-C & p.Lys $1014 \operatorname{Arg} f s^{*} 20$ & Frameshift & $4.01 \mathrm{E}-06$ \\
\hline 20-9449228-G-T & p.Glu1075* & Stop gained & $3.98 \mathrm{E}-06$ \\
\hline 20-9449234-C-T & p.Arg $1077^{*}$ & Stop gained & $3.98 \mathrm{E}-06$ \\
\hline 20-9453451-TAAAC-T & p.Asn1111Ala $f s^{*} 16$ & Frameshift & $4.07 \mathrm{E}-06$ \\
\hline 20-9453986-G-T & p.Glu1145* & Stop gained & $4.09 \mathrm{E}-06$ \\
\hline 20-9459570-G-T & p.Glu1167* & Stop gained & $7.96 \mathrm{E}-06$ \\
\hline 20-9459583-C-T & p.Gln1159* & Stop gained & $3.98 \mathrm{E}-06$ \\
\hline
\end{tabular}

of alterations in a healthy cohort (Table 2). The above findings support the genetic concept of a negative dominance phenomenon according to which a mutated polypeptide with a missense alteration reduces the activity of co-expressed wild-type protein. Therefore, only the suppression of the two PLCB4 copies resulting either from truncating mutation on both alleles or dominant-negative effect exerted by a heterozygous missense variant on the wild-type allele would give rise to ACS2 phenotype. The main limitation of all PLCB4 studies, however, seems to be the lack of functional analyses, which would allow for a better understanding of the data described above.

Interestingly, we have found that one out of thirteen missense mutations - c.1861C > T p.Arg621Cys, which was described in an affected individual M003 and his unaffected father, also occurs in a healthy cohort with a frequency of 0.00031 . The data come from the genome sequencing, whereas the variant was noted among the African population. According to our in-house WES data, the variant was not present in 100 Polish controls, similarly to 1200 control exomes, filtered by Rieder et al. (2012). The gnomAD data associated with the presence of the unaffected carrier, however, may underpin the hypothesis of p.Arg621Cys incomplete penetrance.

To conclude, with this report, we have increased the total number of all molecularly confirmed ACS cases to 30 . Noteworthy, the majority of those patients carry pathogenic variants within the PLCB4 gene that cause ACS2. As mentioned above, the syndrome presents high phenotypic variability among and between affected families that impedes reaching an exact and unequivocal clinical and molecular diagnosis. Thus, describing additional clinically and molecularly well-characterized cases with variable phenotypes, even if carrying previously reported mutations, is essential for the increase of our knowledge in such orphan Mendelian diseases.
Acknowledgments We are grateful to the patient and his parents for participating in this study. We also thank Prof. John Sondek from the Department of Pharmacology, the University of North Carolina at Chapel Hill, Chapel Hill, USA, for his valuable comments regarding PLCB4 modeling.

Authors' contributions E.B.-O. performed targeted next-generation sequencing and Sanger sequencing analysis and wrote the manuscript; E.B.-O., A.S.-S., D.P., and J.W.-S prepared figures; E.B.-O. and F.t. designed primers and prepared tables and supplementary materials; A.J. designed the study, diagnosed the patient clinically, analyzed the WES data, wrote the "Clinical report" section, and critically revised and corrected the manuscript. All authors have read and approved the final manuscript.

Funding This work was supported by the grant from the Polish National Science Centre UMO-2016/22/E/NZ5/00270 to A.J. and by the grant from the Poznan University of Medical Sciences, Poland, 502-1411261860-41259 and from the Polish National Science Centre, Poland, UMO-2016/23/N/NZ5/02577 to E.B.-O.

Data availability The data that support the findings of this study are available from the corresponding author upon reasonable request.

\section{Compliance with ethical standards}

Conflict of interest The authors declare that they have no conflict of interest.

Ethical approval All procedures performed in studies that involved human participants were under the ethical standards of the institutional and national research committee and with the 1964 Helsinki declaration and its later amendments or comparable ethical standards. Ethics approval was granted by the Institutional Review Board of Poznan University of Medical Sciences (no. 783/13 and 741/17).

Consent to participate Informed consent was obtained from all individuals included in the study. The proband and his parents agreed to participate in this study.

Consent for publication The additional consent for publication of identifying information/images in an online open-access publication was also obtained. 
Open Access This article is licensed under a Creative Commons Attribution 4.0 International License, which permits use, sharing, adaptation, distribution and reproduction in any medium or format, as long as you give appropriate credit to the original author(s) and the source, provide a link to the Creative Commons licence, and indicate if changes were made. The images or other third party material in this article are included in the article's Creative Commons licence, unless indicated otherwise in a credit line to the material. If material is not included in the article's Creative Commons licence and your intended use is not permitted by statutory regulation or exceeds the permitted use, you will need to obtain permission directly from the copyright holder. To view a copy of this licence, visit http://creativecommons.org/licenses/by/4.0/.

\section{References}

Bonano M, Tríbulo C, De Calisto J, Marchant L, Sánchez SS, Mayor R, Aybar MJ (2008) A new role for the endothelin-1/endothelin-A receptor signaling during early neural crest specification. Dev Biol 323(1):114-129. https://doi.org/10.1016/j.ydbio.2008.08.007

Bukowska-Olech E, Materna-Kiryluk A, Walczak-Sztulpa J, Popiel D, Badura-Stronka M, Koczyk G, Dawidziuk A, Jamsheer A (2020) Targeted next-generation sequencing in the diagnosis of facial dysostoses. Front Genet. https://doi.org/10.3389/fgene.2020. 580477

Essen LO, Perisic O, M Katan YW, Roberts MF, Williams RL (1997) Structural mapping of the catalytic mechanism for a mammalian phosphoinositide-specific phospholipase C. Biochemistry 36(7): 1704-1718

Gordon CT, Petit F, Kroisel PM, Jakobsen L, Zechi-Ceide RM, Oufadem M, Bole-Feysot C et al (2013a) Mutations in endothelin 1 cause recessive auriculocondylar syndrome and dominant isolated question-mark ears. Am J Hum Genet 93(6):1118-1125. https:// doi.org/10.1016/j.ajhg.2013.10.023

Gordon CT, Vuillot A, Marlin S, Gerkes E, Henderson A, AlKindy A, Holder-Espinasse M et al (2013b) Heterogeneity of Mutational Mechanisms and Modes of Inheritance in Auriculocondylar Syndrome. J Med Genet 50(3):174-186. https://doi.org/10.1136/ jmedgenet-2012-101331

Gordon CT, Nicole Weaver K, Zechi-ceide RM, Madsen EC, Tavares ALP, Oufadem M, Kurihara Y et al (2015) Mutations in the endothelin receptor type A cause mandibulofacial dysostosis with alopecia:519-531. https://doi.org/10.1016/j.ajhg.2015.01.015

Hicks SN, Jezyk MR, Gershburg S, Seifert JP, Harden TK, Sondek J (2008) General and versatile autoinhibition of PLC isozymes. Mol Cell 31(3):383-394. https://doi.org/10.1016/j.molcel.2008.06.018
Jamsheer A, Olech EM, Kozłowski K, Niedziela M, Sowińska-Seidler A, Obara-Moszyńska M, Latos-Bieleńska A, Karczewski M, Zemojtel $\mathrm{T}$ (2016) Exome sequencing reveals two novel compound heterozygous XYLT1 mutations in a Polish patient with Desbuquois dysplasia type 2 and growth hormone deficiency. J Hum Genet 61(7). https://doi.org/10.1038/jhg.2016.30

Kido Y, Gordon CT, Sakazume S, Bdira EB, Dattani M, Wilson LC, Lyonnet $S$ et al (2013) Further characterization of atypical features in auriculocondylar syndrome caused by recessive PLCB4 mutations. Am J Med Genet A 161A(9):2339-2346. https://doi.org/10. 1002/ajmg.a.36066

Leoni C, Gordon CT, Della Marca G, Giorgio V, Onesimo R, Perrino F, Cianfoni A, Cerchiari A, Amiel J, Zampino G (2016) Respiratory and gastrointestinal dysfunctions associated with auriculo-condylar syndrome and a homozygous PLCB4 loss-of-function mutation. Am J Med Genet A 170(6):1471-1478. https://doi.org/10.1002/ ajmg.a.37625

Nabil A, El Shafei S, El Shakankiri NM, Habib A, Morsy H, Maddirevula S, Alkuraya FS (2020) A familial PLCB4 mutation causing auriculocondylar syndrome 2 with variable severity. Eur J Med Genet:103917. https://doi.org/10.1016/j.ejmg.2020.103917

Passos-Bueno MR, Ornelas CC, Fanganiello RD (2009) Syndromes of the first and second pharyngeal arches: a review. Am J Med Genet A 149A(8):1853-1859. https://doi.org/10.1002/ajmg.a.32950

Rieder MJ, Green GE, Park SS, Stamper BD, Gordon CT, Johnson JM, Cunniff CM et al (2012) A human homeotic transformation resulting from mutations in PLCB4 and GNAI3 causes auriculocondylar syndrome. Am J Hum Genet 90(5):907-914. https://doi.org/10.1016/j.ajhg.2012.04.002

Romanelli Tavares VL, Zechi-Ceide RM, Bertola DR, Gordon CT, Ferreira SG, Hsia GSP, Yamamoto GL et al (2017) Targeted molecular investigation in patients within the clinical spectrum of auriculocondylar syndrome. Am J Med Genet A 173(4):938-945. https://doi.org/10.1002/ajmg.a.38101

Storm AL, Johnson JM, Lammer E, Green GE, Cunniff C (2005) Auriculo-condylar syndrome is associated with highly variable ear and mandibular defects in multiple kindreds. Am J Med Genet A 138A(2):141-145. https://doi.org/10.1002/ajmg.a.30883

Waterhouse A, Bertoni M, Bienert S, Studer G, Tauriello G, Gumienny R, Heer FT et al (2018) SWISS-MODEL: homology modelling of protein structures and complexes. Nucleic Acids Res 46(W1): W296-W303. https://doi.org/10.1093/nar/gky427

Publisher's note Springer Nature remains neutral with regard to jurisdictional claims in published maps and institutional affiliations. 